\title{
Enhanced Telecom Emission from Single Group-IV Quantum Dots by Precise CMOS-Compatible Positioning in Photonic Crystal Cavities
}

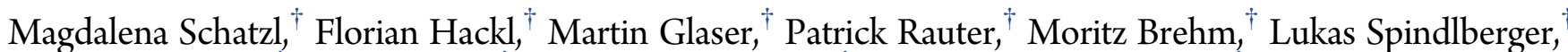
Angelica Simbula, ${ }^{\ddagger}$ Matteo Galli, ${ }^{\ddagger}$ Thomas Fromherz, ${ }^{* \dagger}+{ }^{\dagger}$ and Friedrich Schäffler

${ }^{\dagger}$ Institute of Semiconductor and Solid State Physics, Johannes Kepler University Linz, Altenbergerstraße 69, 4040 Linz, Austria

${ }^{\ddagger}$ Dipartimento di Fisica, Università degli Studi di Pavia, Via A. Bassi 6, 27100 Pavia, Italy

\section{Supporting Information}

ABSTRACT: Efficient coupling to integrated high-quality-factor cavities is crucial for the employment of germanium quantum dot (QD) emitters in future monolithic silicon-based optoelectronic platforms. We report on strongly enhanced emission from single $\mathrm{Ge}$ QDs into L3 photonic crystal resonator (PCR) modes based on precise positioning of these dots at the maximum of the respective mode field energy density. Perfect site control of Ge QDs grown on prepatterned silicon-on-insulator substrates was exploited to fabricate in one processing run almost 300 PCRs containing single QDs in systematically varying positions within the cavities. Extensive photoluminescence

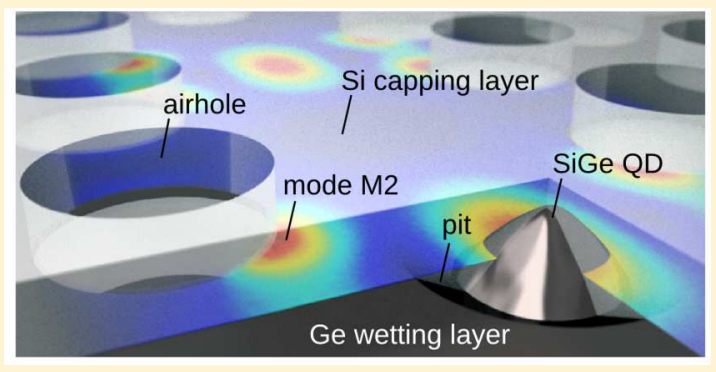
studies on this cavity chip enable a direct evaluation of the positiondependent coupling efficiency between single dots and selected cavity modes. The experimental results demonstrate the great potential of the approach allowing CMOS-compatible parallel fabrication of arrays of spatially matched dot/cavity systems for group-IV-based data transfer or quantum optical systems in the telecom regime.

KEYWORDS: photonic crystal cavities, Purcell effect, position-controlled germanium quantum dots, silicon photonics

ver the last years the prospects of monolithically integrated optical interconnects ${ }^{1}$ as well as on-chip sensing systems ${ }^{2,3}$ and quantum optical platforms ${ }^{4,5}$ have triggered the development of $\mathrm{Si}$-based optical components $^{6-12}$ and sources based on group-IV emitters ${ }^{13-18}$ or hybrid integration of III-V materials., ${ }^{49-22}$ Despite the progress of direct epitaxial growth of III-V semiconductors on $\mathrm{Si}^{20-22}$ group-IV quantum dots (QDs) distinguish themselves as particularly promising candidates for the next generation of sources for Si integrated quantum optical applications. Among their main advantages over III-V-based emitters are their emission in the telecom band as well as the availability of $\mathrm{Ge}$ epitaxial processes in standard CMOS production lines suitable for the monolithic integration of Ge QDs with optoelectronic Si chips. Embedding of Ge QDs in a photonic crystal slab (PCS) or resonator (PCR) for emission enhancement in the 1.3-1.5 $\mu \mathrm{m}$ telecommunication range has recently been demonstrated. $^{23-25}$ For the efficient coupling of QD emission to PCS or PCR modes, the precise position of the dot relative to the two-dimensional mode pattern of the respective photonic crystal (PC) structure is crucial. Nevertheless, most efforts in the group-IV system up to now were based on randomly nucleated Ge QDs without any control of the dot position. $^{23-25}$ Efficient coupling to PCR modes is particularly important in the case of single-dot emitters for quantum optical applications, where source implementations in the III-V system are commonly based on the fabrication of a dedicated PCR around a singled-out randomly nucleated QD. ${ }^{26}$ In contrast to the III-V system, where uniform site-controlled growth of single QDs remains challenging, ${ }^{27-29}$ a robust technological basis for precise positioning of size-homogeneous Ge QDs on pit-patterned substrates has recently been established. ${ }^{30-32}$

Very recently, the integration of site-controlled single $\mathrm{Ge}$ QDs into $\mathrm{H}^{33}$ and $\mathrm{L}^{34}$ PCRs has been reported. However, the emission enhancement of a single group-IV QD in a CMOS-compatible PCR has not yet been directly demonstrated, as the emission from such a system was up to now only compared to the intensity emitted by a reference ensemble of QDs, ${ }^{33}$ or even to an ensemble null-emission in the case of ref 34. As a consequence, the expected influence of the precise QD position on the PCR-induced emission enhancement remains experimentally unexplored for the site-controlled Ge QD platform. In this contribution, we unambiguously demonstrate photoluminescence (PL) emission enhancement for single Ge QDs embedded in L3 PCRs. For this purpose, we studied the dependence of the PL intensity on the precise dot position in the cavity, spectrally resolved for a series of resonator modes. The comparison between the PL emission from a single QD positioned at a local electric field maximum of a targeted PCR mode and a dot located at a field node shows an intensity contrast of multiple orders of magnitude. The parallel

Received: December 29, 2016

Published: February 13, 2017 


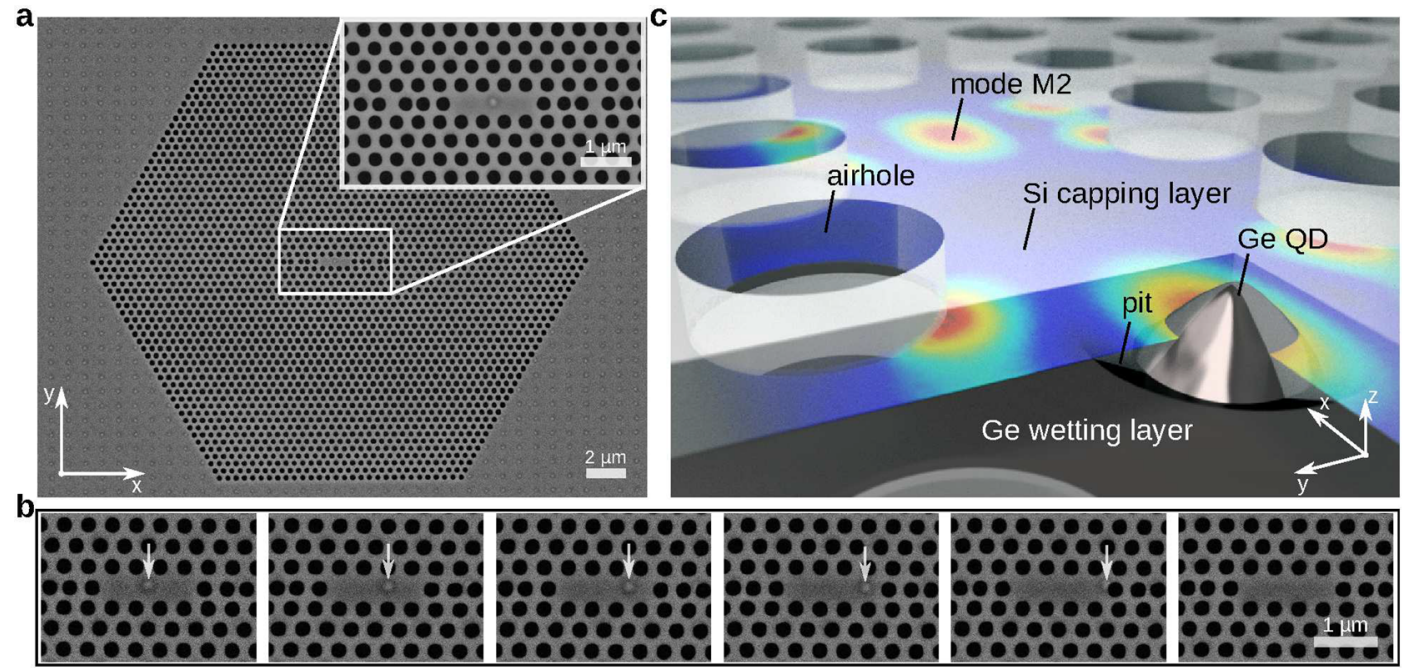

Figure 1. Layout of the single-dot photonic crystal cavities. (a) Scanning electron micrograph of a complete PCR structure with a single Ge QD in the center of the L3 cavity (inset), fabricated in one growth and processing run. The QD array with twice the period of the PC array remains only outside the photonic structure. The inset reveals the modifications of the air hole positions adjacent to the cavity of our adapted high- $Q$ design. (b) Representative set of six simultaneously fabricated L3 cavities in which the Ge QD position (marked by an arrow) was varied along the horizontal center line of the cavity. As a reference, one of the cavities was fabricated without a pit for QD nucleation; that is, it contained only the Ge wetting layer (last frame). (c) Schematic view (not to scale) of a single QD positioned in the calculated field energy density maximum of the M2 cavity mode. The structural components of the single-dot emitter system are indicated. Note that the dot position is determined by the pit in the prepatterned substrate, as described in the Methods section. The displayed dot shape was modeled on an atomic force micrograph.

processing of a series of single-dot cavities with gradually varying dot position allows us to tune the coupling efficiency between the QD and a number of different cavity modes. Therefore, our work not only gives a clear demonstration of enhanced emission from single group-IV QDs in a silicon PCR, it also shows the vast potential of the employed dot positioning approach in terms of parallel processing of large arrays of tailored single-dot cavity emitters for future CMOS-compatible quantum optical platforms.

\section{RESULTS AND DISCUSSION}

Photonic Crystal Cavity Layout. For our work on cavity enhancement of single-dot emission, a large number of PCRs in an L3 geometry ${ }^{35}$ were realized on a silicon chip. For a fixed Si slab thickness $h=220 \mathrm{~nm}$ on top of a $2000 \mathrm{~nm}$ buried $\mathrm{SiO}_{2}$ layer, the PC periodicity $a$ was varied in seven steps between 330 and $420 \mathrm{~nm}$, and each period was implemented with four different hole radii to cover a range of filling factors $r / a$ from 0.305 to 0.325 . Variations of both $a$ and $r / a$ shift the energetic positions of the cavity modes ${ }^{36}$ and thus allow for an adjustment of the spectral overlap between the PCR modes and the emission range of the single QD in each cavity (see Figure S3, Supporting Information). In each of the geometrically defined PCR sets the QD position was systematically shifted in nine steps throughout one-half of the L3 cavity along the long cavity axis ( $x$-direction). As a reference, one cavity in each series contains only the Ge wetting layer (WL), but no QD.

Sample Fabrication. The arrays of PCRs with embedded single Ge QDs were fabricated on silicon-on-insulator (SOI) substrates in a modified version of our recently developed processing sequence ${ }^{33,37}$ as described in the Methods section as well as in Figure S2 of the Supporting Information. Alongside a planarized PCR array chip for optical characterization, a conformally Si-capped but otherwise identical sample was fabricated for imaging purposes. Figure $1 \mathrm{a}, \mathrm{b}$ show representa- tive electron micrographs of fabricated L3 cavities, illustrating the overall PCR layout as well as the parallel fabrication of equivalent cavities with varying single-dot positions. Figure 1c schematically illustrates the detailed three-dimensional structure of a PCR with embedded QD.

Optical Characterization. The emission by single Ge QDs embedded in L3 PCRs was characterized in photoluminescence experiments at a temperature of $10 \mathrm{~K}$ as described in the Methods section. The origin of the studied PL from single QDs was ensured by eliminating potential spurious defect PL and was confirmed in meticulous control experiments (see Methods section). By recording a series of spectrally resolved PL characteristics from the different PCRs on our chip, the cavities featuring optimal spectral mode overlap with the embedded QDs were systematically determined (see Figure S3, Supporting Information). For the high-quality (Q)-factor modes (M0, M1 with $Q>10000)$ and the low- $Q$-mode group (M2-M4, $Q$ $<3000)$ two optimal PCR sets were identified with $a=357 \mathrm{~nm}$ and $a=378 \mathrm{~nm}$, respectively, and $r / a=0.31$ (simulation results for the PCR modes M0-M4 given in Section I of the Supporting Information). Note that due to the prepatterned growth approach, our Ge QDs are very homogeneous with respect to composition profile and size ${ }^{38}$ and exhibit a broad time-averaged emission spectrum typical for the material system $^{39}$ and the employed pumping intensity. Therefore, the emission spectra of the QDs on our PCR chip are comparable under the given experimental conditions, and the time-averaged spectral width of each dot is large enough to include all cavity modes of the selected high- or low- $Q$ group. All of this is in contrast to dots in the III-V system, where achieving efficient coupling between a QD and a PCR mode requires meticulous spectral matching by spectral precharacterization of an individual $\mathrm{QD}$ and subsequent fabrication of a dedicated PC cavity. Furthermore, while prepatterned growth of III-V QDs generally degrades the dot performance as compared to random nucleation, ${ }^{27-29}$ position-controlled Ge dot growth 
leads to greatly improved emission characteristics in terms of spectral dot-to-dot homogeneity while preserving the quantum efficiency. ${ }^{38,40}$ Note that the spectral broadening observed for the single QDs studied here is with high certainty inhomogeneous and caused by spectral diffusion ${ }^{39,41}$ (see Supporting Information, Section IV). Therefore, the spectral width does not pose a principle obstacle for the use of the dots as quantum optical sources.

Figure 2a shows PL spectra acquired for a series of different dot positions in a cavity with $a=357 \mathrm{~nm}$ and $r / a=0.31$. In the
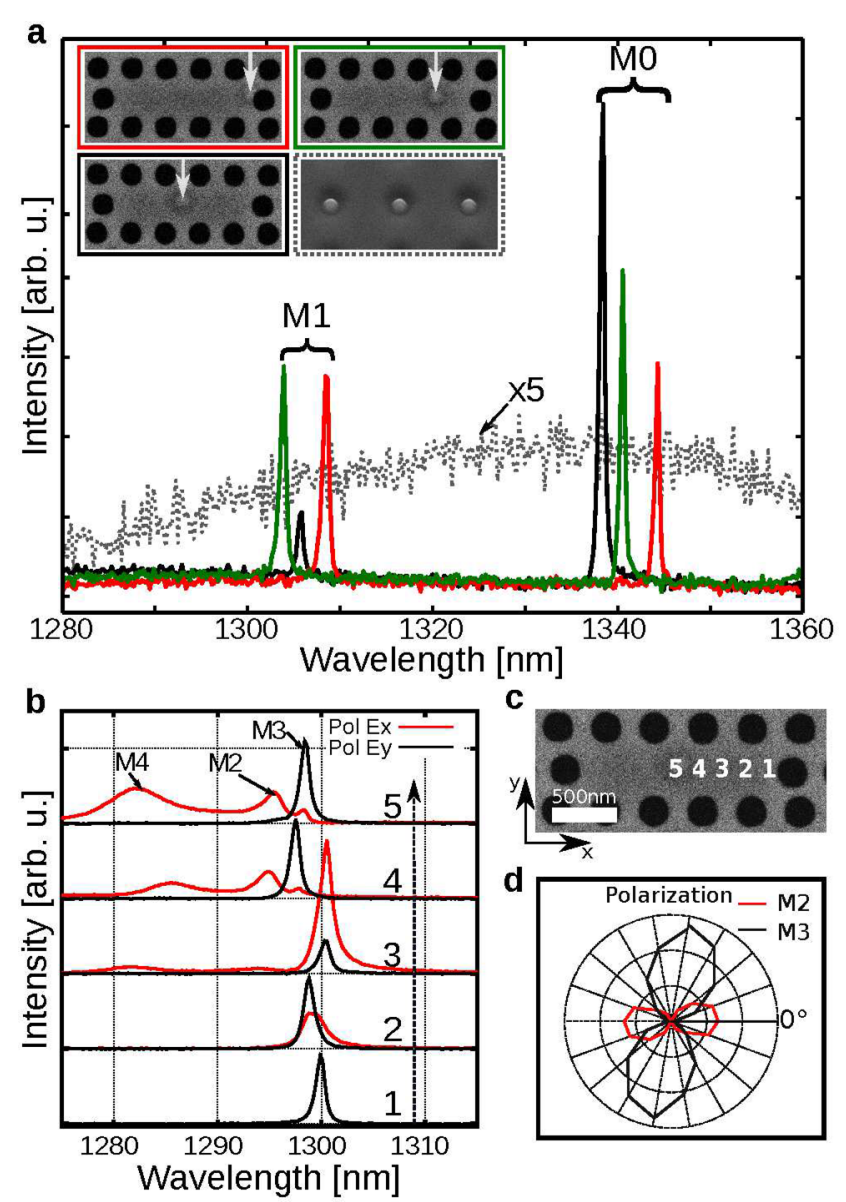

Figure 2. Photoluminescence spectra for a series of single quantum dots at varying positions within the photonic crystal cavity. (a) PL spectra of cavity modes M0 and M1 for the cavity parameters $a=357$ $\mathrm{nm}, r / a=0.31$, and the QD positions indicated in the insets (black: center, red: edge, green: between center and edge); the reference spectrum (broken line) was obtained for an ensemble of ordered QDs from a region outside the PCRs (see Figure 1a), scaled up by a factor of 5 for better visibility. (b) Modes M2-M4 for the five QD positions $1-5$ [indicated in (c)] in an L3 cavity of $a=378 \mathrm{~nm}$ and $r / a=0.31$; two orthogonal polarization components are shown, where the $x$ direction is parallel to the long L3 cavity axis. (d) The orthogonal polarization states of modes M2 and M3 are highlighted in a polar plot. The different PCR periods for (a) and (b) were chosen to align the selected cavity modes to the QD emission band. All experiments were performed at a temperature of $10 \mathrm{~K}$.

presented spectral region, the single dots emit into two cavity modes identified as the M0 and M1 high- $Q$ modes of the PCR, which holds true not only for the representatively shown three dots but for QDs in all of the nine different positions within equivalent cavities on the chip. This observation already clearly indicates a spectral overlap between the PCR modes and the respective QD in all cases and therefore a broad but uniform QD emission across the fabricated chip on the experimental time scale (integration times on the order of seconds) and for high pumping intensity. The broken line represents the emission from an ensemble of arrayed QDs embedded in a chip region without PC structuring, demonstrating that the emission from single QDs in a cavity covers a comparable spectral region with the ensemble emission. Note that the peaks in the cavity spectra shift slightly with the dot position, which is due to a dot-induced change in the effective refractive index of the PCR modes as predicted by the simulations. A change in the PCR parameters allows the shifting of a different set of cavity modes into the time-averaged broad $\mathrm{QD}$ emission region, as shown in Figure $2 \mathrm{~b}$ for cavities with $a=378 \mathrm{~nm}$ and $r / a=0.31$. As indicated by the emission spectra, the five QDs embedded at different positions within equivalent cavities (see Figure 2c) couple to the three low- $Q$ modes M2 to M4 with different strength. In the plot, intensities for the two orthogonal in-plane electric field components are shown, demonstrating that M3 is orthogonally polarized to M2 and M4. The polarization characteristics of the M2 and M3 modes are further highlighted in the polar plot of Figure $2 \mathrm{~d}$ and are consistent with the well-known polarization states of L3-cavity modes (see Figure S1, Supporting Information). A rough but reliable lower limit estimation of the Purcell factor ${ }^{42}\left(F_{\mathrm{P}}\right)$ based on the data in Figure 2 yields $F_{\mathrm{P}} \approx 30$. Details on this estimation are given in section III of the Supporting Information.

While the data shown in Figure 2 clearly indicate that a series of single QDs placed at different positions in equivalent PCRs couple to the same cavity modes with varying efficiency, it does not intuitively reveal a correlation between the QD position and the coupling strength of the dot with resonator modes. Such a dependence, however, does become evident from the full parameter set of emission data from single QDs at nine varying positions within PCRs of two different layouts: Figure 3 shows the integral PL intensity emitted into PCR modes M0 to M4 for a set of QDs as a function of their $x$-position relative to the cavity center. While nine positions within the PCR are covered by the fabricated chip, the plotted data in Figure 3 were mirrored at the cavity center to present a more intuitive picture. Within each panel, the displayed data points (connected red dots) were measured with a constant excitation laser power on cavities with identical geometrical parameters. The shown PL intensities $\left(I_{\mathrm{PL}}\right)$ are integrated over the resonance line width and normalized to the maximum value of $I_{\mathrm{PL}}$ in the respective panel. For the groups of high- $Q$ and low- $Q$ modes the same geometry parameters were employed as in Figure $2 \mathrm{a}$ and $\mathrm{b}$, respectively.

As seen in the plot, the recorded emission into a selected cavity mode varies systematically with the emitter position. This spatial dependence becomes obvious when comparing the PL data to the field energy density patterns of the five cavity modes. For that purpose, the color maps in Figure 3 present the electric field energy density maps for the L3 cavity modes M0 to M4 gained from our simulations. The gray-shaded areas in the PL plots represent a central line cut through these intensity maps, revealing a good reproduction of the spatial mode intensity profiles by the position-dependent integral PL data. This observation clearly indicates that the emission intensity into the respective cavity modes for each QD dominantly depends on the precise dot position within the resonator and is only secondarily influenced by variations in the dot properties 

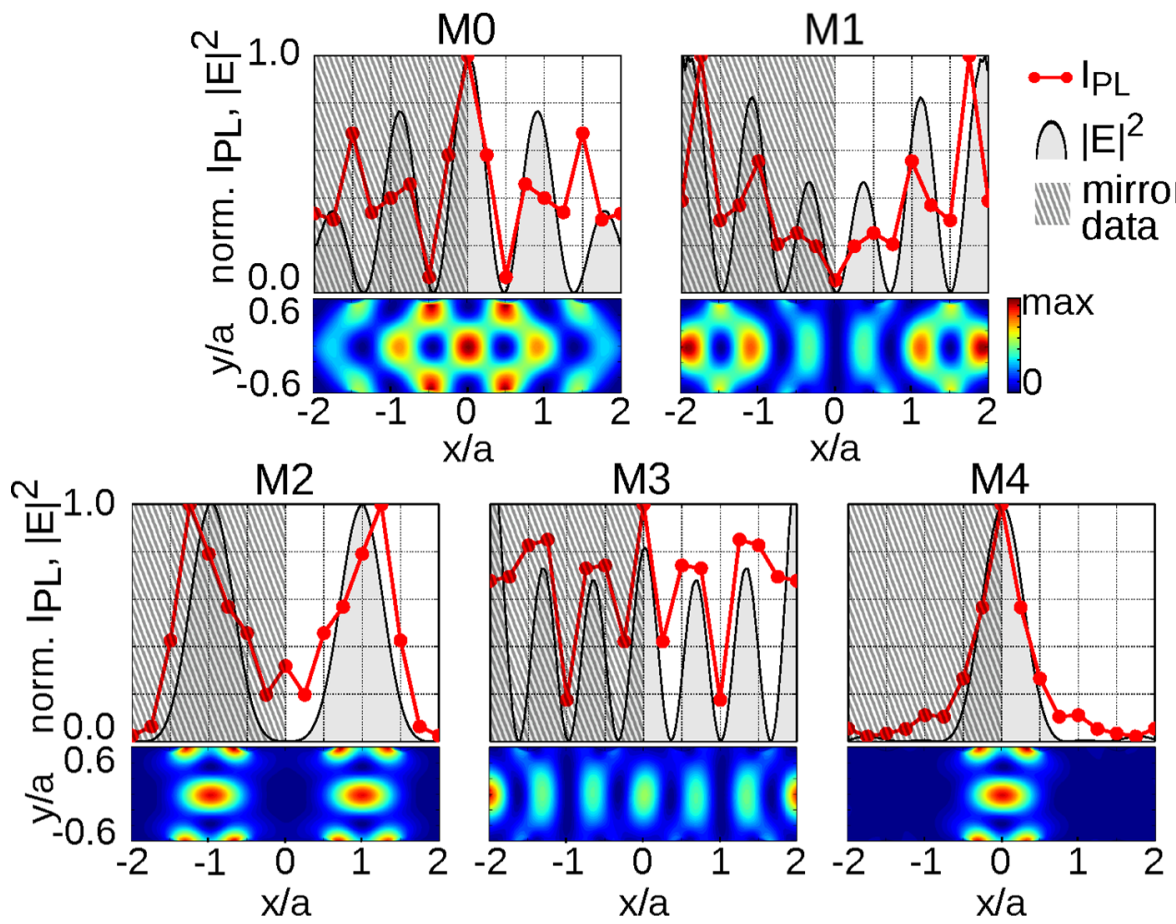

Figure 3. Position-dependent dot emission intensity for different cavity modes. For a series of single dots, the integrated PL intensities $I_{\mathrm{PL}}$ are shown by the red dots for PCR modes M0 to M4 in dependence on the dot position within the cavity. The color maps display the electric field energy distribution $|E|^{2}(x, y)$ within the L3 cavity for each mode up to the edges of the first air holes in both the $x$ - and $y$-directions. Line scans through the center of these simulated maps (gray areas in the $I_{\mathrm{PL}}$ plots) reproduce the experimental position-dependent $I_{\mathrm{PL}}$ data remarkably well. This clearly demonstrates the strong dependence of the coupling efficiency between single QDs and individual PCR modes on the precise dot position. The upper (lower) row of $I_{\mathrm{PL}}$ data was recorded on cavities with a PCS period of $a=357 \mathrm{~nm}(378 \mathrm{~nm})$ and $r / a=0.31$ and represents high- (low-) $Q$ modes M0 and M1 (M2-M4). Note that the shaded negative half-space of the plot is a mirrored reproduction of the data measured for a series of nine dots. All traces are normalized to their respective maximum. As discussed in the Methods section, we estimate the relative standard deviations $\Delta I_{\mathrm{PL}} / I_{\mathrm{PL}}$ to be 0.2 and 0.17 for $\mathrm{M} 0$ and $\mathrm{M} 1$, respectively, and as 0.1 for $\mathrm{M} 2-\mathrm{M} 4$.

across the fabricated chip. Put differently, the inhomogeneously broadened emission of our Ge QDs relaxes the conditions for spectral matching between $\mathrm{QD}$ transitions and PCR modes to a degree, where a series of QDs show virtually equivalent spectral properties. Efficient coupling to a selected mode then only requires spatial matching between dot and cavity. And while this holds true only under certain experimental conditions such as long data acquisition times and high pumping intensities, the data in Figure 3 serve as an unambiguous demonstration of the influence of the single QD position on the coupling efficiency to a series of cavity modes. This is of course also of relevance for cases in which additional spectral matching is required.

\section{DISCUSSION AND CONCLUSION}

As discussed in the Methods section, the interpretation of our experimental results is based on the solid assumption that the studied cavity-QD system operates in the so-called bad cavity limit. ${ }^{43}$ In this limit, the enhancement of the spontaneous emission rate of a single QD in an optical cavity is commonly quantified by the Purcell factor, $F_{\mathrm{P}},{ }^{42,44}$ which is defined as the ratio between the radiative free-decay lifetime $\left(\tau_{\text {free }}\right)$ and the respective cavity decay time $\left(\tau_{\mathrm{c}}\right)$. In a generalized form $F_{\mathrm{P}}$ depends on both the spatial and the spectral overlap between cavity and emitter according to ${ }^{45}$

$$
F_{\mathrm{P}}\left(\mathbf{r}, \omega_{\mathrm{e}}, \hat{e}_{\mathrm{e}}\right)=\frac{\tau_{\text {free }}}{\tau_{\mathrm{c}}}=F_{\mathrm{P}}^{\max } \frac{\kappa^{2}}{4\left(\omega_{\mathrm{e}}-\omega_{\mathrm{c}}\right)^{2}+\kappa^{2}} \frac{\left|\mathbf{E}\left(\mathbf{r}_{\mathrm{e}}\right)\right|^{2}}{\left|E_{\max }\right|^{2}}\left|\hat{e}_{\mathrm{e}} \hat{e}_{\mathrm{c}}\right|^{2}
$$

The first term on the right-hand side is the maximum Purcell factor $F_{\mathrm{P}}^{\mathrm{max}}=3 Q\left(\lambda_{\mathrm{c}} / n\right)^{3} /\left(4 \pi^{2} V_{\text {eff }}\right)$ with the cavity quality factor $Q$ the resonance wavelength $\lambda_{\mathrm{c}} / n$ in a cavity medium with an index of refraction $n$, and the effective mode volume $V_{\text {eff. The }}$ second term defines the spectral overlap of a dipole emitter of frequency $\omega_{\mathrm{e}}$ with cavity mode $\omega_{\mathcal{c}}$ where $\kappa=Q / \omega_{\mathrm{c}}$. The third term gives the spatial overlap expressed in terms of the modal electric field strength $\mathbf{E}\left(\mathbf{r}_{\mathrm{e}}\right)$ at the emitter position $\mathbf{r}_{\mathrm{e}}$ and the mode's maximum modulus of the electric field $E_{\max }$. The fourth term contains the polarization unit vectors of emitter and cavity.

The experimental method presented in the previous section is based on a comparison of the measured PL emission intensity $I_{\mathrm{PL}}$ for a series of QDs placed at different cavity positions. Now, in the bad-cavity regime $I_{\mathrm{PL}}$ scales with $F_{\mathrm{P}}$. Therefore, relating the spectrally resolved $I_{\mathrm{PL}}$ of a series of QDs in a cavity is equivalent to a comparison of the related $F_{\mathrm{P}}$ values. A linear dependence between $I_{\mathrm{PL}}$ and $F_{\mathrm{P}}$ is given only if the overall relaxation time of the excited dot system is not significantly influenced by the enhanced radiative process. $I_{\mathrm{PL}}$ is generally proportional to the quantum efficiency $\eta=\tau_{\mathrm{nr}} /\left(\tau_{\mathrm{nr}}+\right.$ $\left.\tau_{\mathrm{c}}\right)$, where $\tau_{\mathrm{nr}}$ is the nonradiative relaxation time, and therefore saturates in the case of very fast radiative processes. Figure 3 compares the spectrally resolved $I_{\mathrm{PL}}$ of single QDs placed in varying cavity positions by displaying the measured data as a function of the dot position. The dot PL data reproduce the electric field energy profiles of the respective simulated PCR modes remarkably well, where the latter are equivalent to $\left|\mathrm{E}\left(\mathbf{r}_{\mathrm{e}}\right)\right|^{2} /\left|E_{\max }\right|^{2}$. Therefore, $I_{\mathrm{PL}}$ is in good approximation 
proportional to $\left|\mathbf{E}\left(\mathbf{r}_{\mathrm{e}}\right)\right|^{2} /\left|E_{\max }\right|^{2}$ for the studied single-dot system under our experimental conditions.

Two conclusions can be drawn from this experimental observation: First, the overall carrier relaxation times of the studied QDs are comparable, and no prominent saturation effects of $I_{\mathrm{PL}}$ due to dominating radiative relaxation processes are observed. Note that the latter would only influence the detailed profile shape of the position-dependent PL intensity, but would not change the position of minima and maxima. Put differently, time-resolved PL experiments are not sensitive to the Purcell enhancement of the radiative relaxation rates for our QDs. Second, the spectral overlap term $\kappa^{2} /\left[4\left(\omega_{\mathrm{e}}-\omega_{\mathrm{c}}\right)^{2}+\kappa^{2}\right]$ in eq 1 does not significantly influence the measured $I_{\mathrm{PL}}$ either, indicating that the studied quantum dots feature a broad and virtually identical free emission spectrum on the experimental time scale. While the origin of the broad single dot emission spectra has not yet been unambiguously identified, strong indication that the radiative transition is inhomogeneously broadened already for a single quantum dot is found in the literature, $^{39,41}$ as briefly reviewed in Section IV of the Supporting Information. The main discrepancies between experimental data and simulations occur for modes that have their energy density maximum at the very edges of the cavity, in particular modes M1 and M3. The edge position is in fact a critical location for a QD, as can be seen in Figure 1b. Because of its finite size, parts of the QD are removed by air-hole etching. Such a damaged QD with one flank terminated by the nonpassivated surface of the air hole cannot be assumed to behave in the same way as an emitter further inside the cavity that is fully embedded in the Si matrix. Another source of deviations between the experimental map and the simulation is the slight misalignment of the dots in the $y$-direction as shown in Figure $1 \mathrm{~b}$ for the uncapped reference structures, which particularly affects the mapping of the M0 edge peak. On the basis of the simulated M0 mode pattern, we estimate an uncertainty of \pm 0.1 for the normalized $I_{\mathrm{PL}}$ due to this effect. The demonstrated one-dimensional mapping of $F_{\mathrm{P}}$ relates to mapping of the optical local density of states as performed in a recently published effort ${ }^{46}$ employing fluorophore-tagged DNA origami.

Figure 3 unambiguously demonstrates that our fabrication approach allows positioning of a single Ge QD in the absolute field maximum of a selected cavity mode and clearly shows that dot placement with an accuracy of at least $a / 4 \approx 90 \mathrm{~nm}$ is crucial for achieving efficient coupling to L3 cavity modes in a PC with periodicity $a$. Already for the relatively large Ge QDs employed in our studies, the emission intensity of the dot increases by at least 1 order of magnitude when shifting the dot position from a field minimum of a mode into its maximum. Our deterministic positioning approach therefore enables spatial matching between a single $\mathrm{QD}$ and a selected PC cavity mode. Furthermore, in the case of the diffusively broadened time-averaged emission spectra of the Ge QDs, the precise dot position can be utilized for spatial mode selection. This is clearly demonstrated in Figure 2b, where a central position within the cavity supports coupling to modes M2, M3, and M4, while the dot emission can be channeled into mode M3 by shifting the dot into the resonator edge position. The future realization of monolithically integrated group-IV singlephoton sources will most likely require a reduction of the temporal fluctuation broadening of the dot transition by stabilizing the charge carrier environment around the dot. The related more stringent requirements for spectral matching between dots and PCR can be addressed in our approach by the fabrication of large cavity arrays. The presented positioning method is therefore a versatile and powerful approach for maximizing the coupling efficiency between single Ge QDs and PCRs, supporting the reliable parallel processing of vast arrays of single-dot emitters for future group-IV single-photon sources.

To summarize, the efficient coupling to optical resonators is key for the realization of telecommunication wavelength sources for monolithically integrated conventional or quantum optical platforms based on group-IV QDs. The actual emission enhancement achieved is crucially dependent on the precise position of the QD emitter within the PC slab or cavity. Our work demonstrates a CMOS-compatible, scalable, and parallel approach for precise and reliable placement of Ge QDs in PCRs. Moreover, the dot-positioning approach based on $\mathrm{Ge}$ QD growth on prepatterned substrates preserves and in terms of spectral homogeneity even improves the dot emission performance, in strong contrast to comparable techniques in the III-V system. The presented PL data recorded on a chip carrying a large number of single-dot PCRs clearly show the crucial influence of the dot position on the coupling efficiency to L3 cavity modes. The experimental results confirm that our approach allows dot placement at the absolute field energy density maximum of a selected mode. An extensive set of PL spectra demonstrates an increase in Purcell enhancement of single-dot emission by more than an order of magnitude through optimizing the dot position. As further shown by our results, a specific mode can be selected by spatial matching between the dot and the cavity mode pattern. Our approach for achieving optimized coupling of telecom QD emitters to PC cavities therefore paves the way toward Si-based single-photon sources for monolithically integrated quantum optical and data transfer systems.

\section{METHODS}

Cavity Design. L3 PCRs are commonly employed for QD emitters in the III-V system, as they support high-Q-factor designs with low mode volumes. The geometry is particularly well suited for the presented study on position-dependent QD emission, as the PC modes are tightly confined to a silicon slab volume free of PC air holes, giving ample space for positionvaried $\mathrm{QD}$ embedding in the silicon bulk material. The general layout of the L3 PCRs for our experiments is loosely based on the design in ref 47 . We adapted the geometry parameters of the reference design to our experimental constraints, in particular the slab thickness $h$, which is mainly defined by the epilayer thickness, and the air-hole radius $r$, which needs to exceed the maximum QD radius in our technology approach (see next section). Therefore, we set $r \geq 75 \mathrm{~nm}$ and $h=220$ $\mathrm{nm}$ as input parameters of finite-difference time-domain (FDTD) simulations in the open source implementation MEEP. ${ }^{48}$ The single Ge QD in the cavity was approximated by a cylinder of radius $r=75 \mathrm{~nm}$, a height $z$ between 20 and 50 $\mathrm{nm}$, and a dielectric constant of $\varepsilon=13.56$. The chosen $\varepsilon$ value corresponds to the average Ge concentration of $\sim 37 \%$ in a typical, site-controlled Ge QD deposited at $700{ }^{\circ} \mathrm{C} .{ }^{49,50}$ The simulated $\mathrm{Ge} \mathrm{QD}$ was located at systematically varying positions along the centerline connecting the three missing air holes that define the L3 cavity ( $x$-direction). In addition, a small indentation on the slab surface directly above the QD was taken into account, which remains even after high-temperature Si capping (see next section) as a residue of the initially etched 
pit for QD nucleation. ${ }^{51}$ The FDTD results revealed that the small amount of higher index material in the QD leads to a slight shift of the cavity modes to higher wavelengths, whereas missing material $(\varepsilon=1)$ above the $\mathrm{QD}$ causes shifts in the opposite direction. Moreover, the respective shifts depend on the location of the QD in the cavity. On the basis of MEEP simulations, systematic changes in the far-field emission profile due to the varying dot position are estimated to influence the experimental collection efficiency of any mode by less than $3 \%$. Figure S1 of the Supporting Information shows the electric field intensity profiles for cavity modes M0 to M4 relative to the PC structure, while Figure 3 compares the spatial mode profile within the L3 cavity to the experimental dot PL data. For the investigated sets of geometry parameters the simulations predict a group of modes with high Q-factors of 42000 and 15000 (modes M0 and M1, respectively), alongside a group of low- $Q$ modes (M2-M4) with values below 3000. Plots of the simulated PCR modes are shown in Figure $\mathrm{S} 1$ of the Supporting Information. On the basis of the simulated mode patterns, we designed mask layouts containing 280 complete PCRs.

Sample Processing. The arrays of PCRs with embedded single Ge QDs were fabricated on custom-made SOI substrates with a $2000 \mathrm{~nm}$ thick buried oxide layer (BOX) on which the manufacturer SOITEC performed an additional thinning/ rebonding/annealing process sequence. ${ }^{52}$ In this way, potential remnants of the hydrogen implantation for the smart-cut process $^{52}$ were removed in a similar way as reported in ref 23 . This substrate preparation process eliminates any potential contributions of optically active defects to the sample PL, which have been recently observed for industrial SOI substrates in the same wavelength range as $\mathrm{SiGe} \mathrm{QD}$ transitions $(1.3-1.6 \mu \mathrm{m})$ even at room temperature. ${ }^{23,53}$ Moreover, the additional thinning process led to a device layer thickness of just 70 $\mathrm{nm}$, which allowed us to vertically center the Ge QDs in the designed slab thickness of $220 \mathrm{~nm}$.

In the first processing step (Figure S2a, Supporting Information), alignment marks are etched deep into the BOX of the custom SOI substrate. They are defined by electron beam lithography (EBL) in a Raith eLine Plus facility equipped with a laser stage that allows for an alignment precision in the $10 \mathrm{~nm}$ range. Anisotropic etching into the BOX is performed at cryogenic temperatures in an inductively coupled (ICP) Oxford 100 reactive ion etcher (RIE) employing an $\mathrm{SF}_{6} / \mathrm{O}_{2}$ process. ${ }^{37}$

In the second step (Figure S2b, Supporting Information), a set of pits for the preferential nucleation of $\mathrm{QDs}^{30,32,54}$ is fabricated by EBL and a shallow ( $35 \pm 5 \mathrm{~nm}$ deep) ICP-RIE process. Complete arrays of pits with twice the periodicity of the air-hole patterns for the PCRs are realized in this lithography level. Only the one pit that defines the QD position in each of the subsequently processed cavities is slightly moved out of registry in a series of 10 adjacent PCR structures. In this way, the cavity QD is shifted systematically between the center and the terminating air hole at one edge of the L3 cavity (Figure 1b). The alignment marks are used for positioning of the pit-pattern mask, thus defining a reference frame that will be employed again for the later PCR fabrication step. Writing complete pit arrays with comparably small periods is essential for tight size control and perfect ordering of the QDs. $^{32}$

In the third processing step (Figure S2c Supporting Information) the epitaxial layers are deposited. The samples are prepared for epitaxy by resist removal in organic solvents and an oxygen-plasma followed by an RCA cleaning procedure. ${ }^{55}$ A final treatment in $1 \%$ hydrofluoric acid (HF) removes the natural oxide and provides a hydrogen-terminated surface that stabilizes the pit pattern during subsequent heat treatments. ${ }^{56}$ The templates are then immediately transferred into the load-lock chamber of a Riber SIVA 45 molecular beam epitaxy system and subsequently degassed in the ultra-highvacuum environment of the growth chamber at $750{ }^{\circ} \mathrm{C}$ for 45 min. The epitaxial layer sequence consists of a $40 \mathrm{~nm}$ thick $\mathrm{Si}$ buffer, $0.66 \mathrm{~nm}$ of Ge, and a $110 \mathrm{~nm}$ thick Si cap. During buffer growth the substrate temperature is ramped from $450{ }^{\circ} \mathrm{C}$ to $550{ }^{\circ} \mathrm{C}$ and is then kept constant at $700{ }^{\circ} \mathrm{C}$ for Ge deposition. $\mathrm{Ge}$ growth leads to an approximately three monolayer thick, Ge-rich WL that covers the entire sample ${ }^{57}$ and arrays of perfectly ordered, dome-shaped Ge QDs, which nucleate at the centers of the predefined pits ${ }^{32}$ (Figure 1a). On average, these QDs are $15 \mathrm{~nm}$ high and have a base diameter of $125 \mathrm{~nm}$. Note that these geometrical dimensions are much larger than those of the confinement volume for electrons and holes. In ref 41 it is shown that the ground-state electrons are confined to the $\mathrm{Si}$ capping layer region with $\sim 20 \mathrm{~nm}$ lateral and $\sim 8 \mathrm{~nm}$ vertical diameter in close vicinity to the $\mathrm{QD}$ apex due to the strain field generated by the QD. Holes in the ground state are confined to the interior of the QD and are also localized close to the apex due to the interplay between Ge concentration gradient and stain field. The localization region for the holes extends laterally $\sim 30 \mathrm{~nm}$ and vertically $\sim 10 \mathrm{~nm}$.

Two versions of the Si capping layer were grown on a pair of samples with otherwise identical growth and layout parameters. For the first one, the substrate temperature was ramped from $350{ }^{\circ} \mathrm{C}$ to $450{ }^{\circ} \mathrm{C}$ to achieve conformal covering of the $\mathrm{Ge}$ QDs. ${ }^{58}$ The second cap version commenced again at $350{ }^{\circ} \mathrm{C}$ to preserve the $\mathrm{QD}$ shape, but was then ramped to $700{ }^{\circ} \mathrm{C}$ to partly planarize the epilayer surface. ${ }^{51}$ The conformally capped samples were mainly used for visualizing the QD positions by SEM (Figure 1). These samples allowed us to validate an overall alignment precision of about $40 \mathrm{~nm}$ for the complete process. The partly planarized samples were used for optical characterization because their more homogeneous slab thickness leads to better defined cavities.

In the last processing step (Figure S2d, Supporting Information) the initially defined alignment marks are exploited again to align the air-hole mask of the PCRs in such a way that the QDs become centered in the air holes. In this way, all QDs are removed during air-hole etching except the one remaining in the cavity. The air holes are ICP-RIE etched with perpendicular sidewalls down to the $\mathrm{BOX}$ in an $\mathrm{SF}_{6} / \mathrm{O}_{2}$ cryoprocess. In a last step, the BOX beneath the PCR structures is selectively removed with $40 \% \mathrm{HF}$ to achieve free-standing PCR slabs.

We want to emphasize that EBL is not essential to our approach and that the required position accuracy is routinely achieved in CMOS processes on an industrial scale. ${ }^{59}$ The technological approach of this work is therefore CMOS compatible.

Cavity Characterization. The $Q$ values of the fabricated cavities were determined by resonant laser scattering experiments, as described in ref 60 . On a Ge-free reference sample, we found $Q=40000$ for mode M0, in excellent agreement with the simulation value for this mode. With the Ge epilayer added, the $Q$-factor of this mode decreased to 8200. The resonant absorption by Ge QDs embedded in SOI PCRs is not expected to significantly affect the $Q$-factor, ${ }^{61}$ and the influence of other 
absorption loss channels (e.g., free carrier absorption) can be excluded in the employed laser scattering characterization scheme as well. A possible cause for the observed $Q$-factor degradation is the enhanced roughness of the air-hole surfaces introduced by the different etching rates for $\mathrm{Si}$ and $\mathrm{Ge}$ in the utilized RIE process. In addition to degrading the $Q$-factors according to $Q^{-1}=Q_{\text {ideal }}^{-1}+Q_{\text {scatter }}^{-1}$, this roughness also induces statistical uncertainty to them. Here $Q_{\text {ideal }}$ denotes the $Q$-factor obtained from simulations, and $Q_{\text {scatter }}$ corresponds to the cavity photon loss rates due to roughness scattering. For our PCR fabrications process, we estimate the statistical standard deviation $\Delta\left(Q_{\text {scatter }}^{-1}\right) \approx 6 \times 10^{-5}$ from the evaluation of the $Q$-factors measured in $\mathrm{PL}$ experiments. Using $\Delta Q / Q=$ $Q \Delta\left(Q^{-1}\right)=Q \Delta\left(Q_{\text {scatter }}^{-1}\right)$, we get 0.2 and 0.17 for the relative, roughness-induced $Q$-factor standard deviations for modes M0 and $\mathrm{M1}$, respectively. In agreement with recently published work $^{61}$ for a similar experimental situation, these values describe also the relative standard deviations $\Delta I_{\mathrm{PL}} / I_{\mathrm{PL}}$ for the profiles shown in the upper panels of Figure 3. For modes M2 to M4, the roughness-induced $Q$-factor uncertainties are smaller than uncertainties in $I_{\mathrm{PL}}$ due to $\mathrm{QD}$ positioning accuracy and varying experimental conditions, which result in $\Delta I_{\mathrm{PL}} / I_{\mathrm{PL}}=0.1$ for the lower panels of Figure 3.

For PCRs containing a Ge layer, the largest Q-factor observed in our resonant scattering experiments is $Q=8200$. This value corresponds to a cavity photon emission rate $\tau_{\mathrm{ph}}=$ $Q \lambda_{\text {res }} /(2 \pi c) \approx 6 \mathrm{ps}$, where $c$ denotes the vacuum speed of light and $\lambda_{\text {res }} \approx 1340 \mathrm{~nm}$ the resonance wavelength of the PCR. As the exciton dephasing time of the studied QDs is expected to be orders of magnitude higher than 6 ps (preliminary data suggests nonradiative exciton lifetimes of tens of ns), the QDcavity system operates with high certainty in the bad-cavity limit.

Photoluminescence Characterization. Photoluminescence measurements were performed with a confocal microPL setup ${ }^{37,54}$ consisting of an excitation diode laser operated at $442 \mathrm{~nm}$ and a microscope objective with a numerical aperture of NA $=0.7$. The latter is used both for laser focusing and for collecting the PL signal from the sample. The detection spot on the sample with a diameter of $\sim 3 \mu \mathrm{m}$ is centered at the selected L3 cavity. At the used excitation wavelength the absorption coefficient of $\mathrm{Si}$ is large enough to allow efficient electron-hole excitation in the $220 \mathrm{~nm}$ thick Si slab enclosing the Ge QDs. For polarization-dependent measurements a $\lambda / 2$ plate and a linear polarizer are used to record spectra in $5^{\circ}$ rotation steps of the former. The signal is collected perpendicular to the sample surface with an acceptance cone covering a solid angle of $0.15 \times$ $4 \pi \mathrm{sr}$. The sample is mounted in a cryostat and maintained at a temperature of $10 \mathrm{~K}$. The signal is dispersed in a grating spectrometer and recorded with a liquid-nitrogen-cooled InGaAs line detector. To enhance spectral overlap between the cavity modes and $\mathrm{QD}$ emission, a rather high laser excitation power of $750 \mu \mathrm{W}$ was chosen, which has been shown to cause significant spectral broadening of the time-averaged emission spectrum of single Ge QDs. ${ }^{39}$

Control Experiments. As discussed in the Sample Processing section, the use of custom-made SOI substrates for our prepatterned growth approach ensured the absence of optically active defects. To confirm the efficient removal of these defects and allow the unambiguous identification of the observed PL intensity as single-dot emission, we performed photoluminescence experiments on both the bare SOI substrates and processed reference samples. The latter underwent all described fabrication steps for L3 cavities with the exception of Ge epilayer growth. Thus, the reference cavities contained all defects that were either caused by SOI substrate fabrication or induced by our subsequent PCR processing sequence. For the reference PCR only negligible indications of defect PL in the spectral range of the Ge QDs were observed, which were $~ 500$ times smaller than the QDrelated signals (see Figure S3d, Supporting Information). Therefore, the PL emission of the PCR structures studied in our work clearly originates from single Ge QDs.

It has to be mentioned that previous work on single Ge QDs in an L3 PCR reported in ref 34 did not include control experiments to exclude spurious $\mathrm{PL}$ from optically active defects, which has been frequently observed in industrial silicon-on-insulator substrates. ${ }^{14,23,53,62}$ Furthermore, no substrate preparation steps targeting the removal of such defects are mentioned in ref 34 . There, the PCR sample is compared to a large reference ensemble of QDs, which does not show any emission, not even at $7 \mathrm{~K}$. In stark contrast, their observed PCR emission persists up to $300 \mathrm{~K}$, which is highly surprising in light of the expected low confinement energies of electrons in $\mathrm{Ge}$ $\mathrm{QDs}^{38,63}$ and hints at spurious contributions from defect PL originating from the SOI substrates. ${ }^{23,53}$

\section{ASSOCIATED CONTENT}

\section{S Supporting Information}

The Supporting Information is available free of charge on the ACS Publications website at DOI: 10.1021/acsphotonics.6b01045.

Simulation of photonic crystal cavity modes; fabrication process; additional photoluminescence data and control experiments; discussion on the origin of broad Ge singledot spectra (PDF)

\section{AUTHOR INFORMATION}

\section{Corresponding Author}

*E-mail: thomas.fromherz@jku.at.

ORCID 8

Thomas Fromherz: 0000-0003-2718-4041

\section{Author Contributions}

M.S. and F.S. designed the research. M.S. designed and fabricated the single-dot cavities and performed all simulations. M.Gl. and M.B. performed the epitaxial growth of the sitecontrolled QDs. M.S., F.H., L.S., and A.S. performed the experiments. M.S., F.H., P.R., M.B., A.S., M.Ga., T.F., and F.S. analyzed and interpreted the data. M.S., P.R., T.F., and F.S. wrote the manuscript. F.S. supervised the project.

\section{Notes}

The authors declare no competing financial interest.

\section{ACKNOWLEDGMENTS}

The authors acknowledge support from the Austrian Science Fund (FWF): F2502-N17, F2512-N17 of the Spezialforschungsbereich IRON (SFB025), P29137-N36, and P28409N36, and the Linz Institute of Technology (LIT): LIT-2016-1YOU-002. We thank G. Katsaros for providing the custommade, rebonded SOI substrates. We are grateful to R. Jannesari, M. Grydlik, and D. Gerace for valuable discussions as well as to A. Halilovic and S. Bräuer for technical clean-room support during the fabrication of the PCRs. 


\section{REFERENCES}

(1) Rickman, A. The commercialization of silicon photonics. Nat. Photonics 2014, 8, 579-582.

(2) Baldassarre, L.; Sakat, E.; Frigerio, J.; Samarelli, A.; Gallacher, K.; Calandrini, E.; Isella, G.; Paul, D. J.; Ortolani, M.; Biagioni, P. Midinfrared Plasmon-Enhanced Spectroscopy with Germanium Antennas on Silicon Substrates. Nano Lett. 2015, 15, 7225-7231.

(3) Juan-Colás, J.; Parkin, A.; Dunn, K. E.; Scullion, M. G.; Krauss, T. F.; Johnson, S. D. The electrophotonic silicon biosensor. Nat. Commun. 2016, 7, 12769.

(4) Chen, Y.; Zhang, J.; Zopf, M.; Jung, K.; Zhang, Y.; Keil, R.; Ding, F.; Schmidt, O. G. Wavelength-tunable entangled photons from silicon-integrated III-V quantum dots. Nat. Commun. 2016, 7, 10387.

(5) Silverstone, J. W.; Bonneau, D.; OBrien, J. L.; Thompson, M. G. Silicon Quantum Photonics. IEEE J. Sel. Top. Quantum Electron. 2016, 22, 6700113.

(6) Chaisakul, P.; Marris-Morini, D.; Frigerio, J.; Chrastina, D.; Rouifed, M.-S.; Cecchi, S.; Crozat, P.; Isella, G.; Vivien, L. Integrated germanium optical interconnects on silicon substrates. Nat. Photonics 2014, 8, 482-488.

(7) Debnath, K.; Gardes, F. Y.; Knights, A. P.; Reed, G. T.; Krauss, T. F.; Ofaolain, L. Dielectric waveguide vertically coupled to all-silicon photodiodes operating at telecommunication wavelengths. Appl. Phys. Lett. 2013, 102, 171106.

(8) Stern, B.; Zhu, X.; Chen, C. P.; Tzuang, L. D.; Cardenas, J.; Bergman, K.; Lipson, M. On-chip mode-division multiplexing switch. Optica 2015, 2, 530-535.

(9) Rouifed, M.-S.; Marris-Morini, D.; Chaisakul, P.; Frigerio, J.; Isella, G.; Chrastina, D.; Edmond, S.; le Roux, X.; Coudevylle, J.-R.; Bouville, D.; Vivien, L. Advances Toward Ge/SiGe Quantum-Well Waveguide Modulators at $1.3 \mu \mathrm{m}$. IEEE J. Sel. Top. Quantum Electron. 2014, 20, 3400207.

(10) Moss, D. J.; Morandotti, R.; Gaeta, A. L.; Lipson, M. New CMOS-compatible platforms based on silicon nitride and Hydex for nonlinear optics. Nat. Photonics 2013, 7, 597-607.

(11) Michel, J.; Liu, J.; Kimerling, L. C. High-performance Ge-on-Si photodetectors. Nat. Photonics 2010, 4, 527-534.

(12) Dumas, D.; Gallacher, K.; Rhead, S.; Myronov, M.; Leadley, D. R.; Paul, D. J. Ge/SiGe quantum confined Stark effect electroabsorption modulation with low voltage swing at $\lambda=1550 \mathrm{~nm}$. Opt. Express 2014, 22, 482-488.

(13) Wirths, S.; Geiger, R.; von den Driesch, N.; Mussler, G.; Stoica, T.; Mantl, S.; Ikonic, Z.; Luysberg, M.; Chiussi, S.; Hartmann, J. M.; Sigg, H.; Faist, J.; Buca, D.; Grützmacher, D. Lasing in direct-bandgap GeSn alloy grown on Si. Nat. Photonics 2015, 9, 88-92.

(14) Shakoor, A.; Lo Savio, R.; Cardile, P.; Portalupi, S. L.; Gerace, D.; Welna, K.; Boninelli, S.; Franzò, G.; Priolo, F.; Krauss, T. F.; Galli, M.; O'Faolain, L. Room temperature all-silicon photonic crystal nanocavity light emitting diode at sub-bandgap wavelengths. Laser Photon. Rev. 2013, 7, 114-121.

(15) Suess, M. J.; Geiger, R.; Minamisawa, R. A.; Schiefler, G.; Frigerio, J.; Chrastina, D.; Isella, G.; Spolenak, R; Faist, J.; Sigg, H. Analysis of enhanced light emission from highly strained germanium microbridges. Nat. Photonics 2013, 7, 466-472.

(16) Grydlik, M.; Hackl, F.; Groiss, H.; Glaser, M.; Halilovic, A.; Fromherz, T.; Jantsch, W.; Schäffler, F.; Brehm, M. Lasing from Glassy Ge Quantum Dots in Crystalline Si. ACS Photonics 2016, 3, 298-303.

(17) Takahashi, Y.; Inui, Y.; Chihara, M.; Asano, T.; Terawaki, R.; Noda, S. A micrometre-scale Raman silicon laser with a microwatt threshold. Nature 2013, 498, 470-474.

(18) Liu, J.; Sun, X.; Camacho-Aguilera, R.; Kimerling, L. C.; Michel, J. Ge-on-Si laser operating at room temperature. Opt. Lett. 2010, 35, 679-681.

(19) Mayer, B.; Janker, L.; Loitsch, B.; Treu, J.; Kostenbader, T.; Lichtmannecker, S.; Reichert, T.; Morkötter, S.; Kaniber, M.; Abstreiter, G.; Gies, C.; Koblmüller, G.; Finley, J. J. Monolithically Integrated High- $\beta$ Nanowire Lasers on Silicon. Nano Lett. 2016, 16, $152-156$.
(20) Chen, S.; Li, W.; Wu, J.; Jiang, Q.; Tang, M.; Shutts, S.; Elliott, S. N.; Sobiesierski, A.; Seeds, A. J.; Ross, I.; Smowton, P. M.; Liu, H. Electrically pumped continuous-wave III-V quantum dot lasers on silicon. Nat. Photonics 2016, 10, 307-311.

(21) Wang, Z.; Tian, B.; Pantouvaki, M.; Guo, W.; Absil, P.; van Campenhout, J.; Merckling, C.; Van Thourhout, D. Room-temperature InP distributed feedback laser array directly grown on silicon. Nat. Photonics 2015, 9, 837-842.

(22) Han, Y.; Li, Q.; Chang, S.-P.; Hsu, W.-D.; Lau, K. M. Growing InGaAs quasi-quantum wires inside semi-rhombic shaped planar InP nanowires on exact (001) silicon. Appl. Phys. Lett. 2016, 108, 242105.

(23) Hauke, N.; Tandaechanurat, A.; Zabel, T.; Reichert, T.; Takagi, H.; Kaniber, M.; Iwamoto, S.; Bougeard, D.; Finley, J.; Abstreiter, G. A three-dimensional silicon photonic crystal nanocavity with enhanced emission from embedded germanium islands. New J. Phys. 2012, 14, 083035 .

(24) Xia, J.; Ikegami, Y.; Shiraki, Y.; Usami, N.; Nakata, Y. Strong resonant luminescence from Ge quantum dots in photonic crystal microcavity at room temperature. Appl. Phys. Lett. 2006, 89, 201102.

(25) Tsuboi, T.; Xu, X.; Xia, J.; Usami, N.; Maruizumi, T.; Shiraki, Y. Room-temperature electroluminescence from $\mathrm{Ge}$ quantum dots embedded in photonic crystal microcavities. Appl. Phys. Express 2012, 5, 052101.

(26) Badolato, A.; Hennessy, K.; Atatüre, M.; Dreiser, J.; Hu, E.; Petroff, P. M.; Imamoğlu, A. Deterministic Coupling of Single Quantum Dots to Single Nanocavity Modes. Science 2005, 308, 11581161.

(27) Schneider, C.; Huggenberger, A.; Sünner, T.; Heindel, T.; Strauß, M.; Göpfert, S.; Weinmann, P.; Reitzenstein, S.; Worschech, L.; Kamp, M.; Höfling, S.; Forchel, A. Single site-controlled In(Ga)As/ GaAs quantum dots: growth, properties and device integration. Nanotechnology 2009, 20, 434012.

(28) Schramm, A.; Tommila, J.; Strelow, C.; Hakkarainen, T. V.; Tukiainen, A.; Dumitrescu, M.; Mews, A.; Kipp, T.; Guina, M. Large array of single, site-controlled InAs quantum dots fabricated by UVnanoimprint lithography and molecular beam epitaxy. Nanotechnology 2012, 23, 175701.

(29) Atkinson, P.; Kiravittaya, S.; Benyoucef, M.; Rastelli, A.; Schmidt, O. G. Site-controlled growth and luminescence of InAs quantum dots using in situ Ga-assisted deoxidation of patterned substrates. Appl. Phys. Lett. 2008, 93, 101908.

(30) Zhong, Z.; Halilovic, A.; Fromherz, T.; Schäffler, F.; Bauer, G. Two-dimensional periodic positioning of self-assembled Ge islands on prepatterned $\mathrm{Si}(001)$ substrates. Appl. Phys. Lett. 2003, 82, 4779.

(31) Stoica, T.; Shushunova, V.; Dais, C.; Solak, H.; Grützmacher, D. Two-dimensional arrays of self-organized Ge islands obtained by chemical vapor deposition on pre-patterned silicon substrates. Nanotechnology 2007, 18, 455307.

(32) Grydlik, M.; Langer, G.; Fromherz, T.; Schäffler, F.; Brehm, M. Recipes for the fabrication of strictly ordered $\mathrm{Ge}$ islands on pitpatterned $\mathrm{Si}(001)$ substrates. Nanotechnology 2013, 24, 105601.

(33) Schatzl, M.; Hackl, F.; Glaser, M.; Fromherz, T.; Schäffler, F. Single $\mathrm{SiGe}$ quantum dots deterministically aligned to the antinodes of a photonic crystal cavity mode. 2015 IEEE 12th International Conference on Group IV Photonics (GFP); 2015; pp 39-40.

(34) Zeng, C.; Ma, Y.; Zhang, Y.; Li, D.; Huang, Z.; Wang, Y.; Huang, Q.; Li, J.; Zhong, Z.; Yu, J.; Jiang, Z.; Xia, J. Single germanium quantum dot embedded in photonic crystal nanocavity for light emitter on silicon chip. Opt. Express 2015, 23, 22250-22261.

(35) Akahane, Y.; Asano, T.; Song, B.-S.; Noda, S. High-Q photonic nanocavity in a two-dimensional photonic crystal. Nature 2003, 425, 944-947.

(36) Joannopoulos, J.; Johnson, S.; Winn, J.; Meade, R. Photonic Crystals: Molding the Flow of Light, 2nd ed.; Princeton University Press, 2011.

(37) Jannesari, R.; Schatzl, M.; Hackl, F.; Glaser, M.; Hingerl, K.; Fromherz, T.; Schäffler, F. Commensurate germanium light emitters in silicon-on-insulator photonic crystal slabs. Opt. Express 2014, 22, 25426-25435. 
(38) Brehm, M.; Grydlik, M.; Hackl, F.; Lausecker, E.; Fromherz, T.; Bauer, G. Excitation Intensity Driven PL Shifts of SiGe Islands on Patterned and Planar Si(001) Substrates: Evidence for Ge-rich Dots in Islands. Nanoscale Res. Lett. 2010, 5, 1868-1872.

(39) Grydlik, M.; Brehm, M.; Tayagaki, T.; Langer, G.; Schmidt, O. G.; Schäffler, F. Optical properties of individual site-controlled Ge quantum dots. Appl. Phys. Lett. 2015, 106, 251904.

(40) Lausecker, E.; Brehm, M.; Grydlik, M.; Hackl, F.; Bergmair, I.; Mühlberger, M.; Fromherz, T.; Schäffler, F.; Bauer, G. UV nanoimprint lithography for the realization of large-area ordered $\mathrm{SiGe} / \mathrm{Si}(001)$ island arrays. Appl. Phys. Lett. 2011, 98, 143101.

(41) Klenovský, P.; Brehm, M.; Křápek, V.; Lausecker, E.; Munzar, D.; Hackl, F.; Steiner, H.; Fromherz, T.; Bauer, G.; Humlíček, J. Excitation intensity dependence of photoluminescence spectra of $\mathrm{SiGe}$ quantum dots grown on prepatterned $\mathrm{Si}$ substrates: Evidence for biexcitonic transition. Phys. Rev. B: Condens. Matter Mater. Phys. 2012, $86,115305$.

(42) Purcell, E. M.; Torrey, H.; Pound, R. V. Resonance absorption by nuclear magnetic moments in a solid. Phys. Rev. 1946, 69, 37.

(43) Rice, P. R.; Carmichael, H. J. Single-atom cavity-enhanced absorption I: Photon statistics in the bad-cavity limit. IEEE J. Quantum Electron. 1988, 24, 1351-1366.

(44) Koenderink, A. F. On the use of Purcell factors for plasmon antennas. Opt. Lett. 2010, 35, 4208-4210.

(45) Gerard, J.-M.; Gayral, B. Strong Purcell effect for InAs quantum boxes in three-dimensional solid-state microcavities. J. Lightwave Technol. 1999, 17, 2089-2095.

(46) Gopinath, A.; Miyazono, E.; Faraon, A.; Rothemund, P. W. K. Engineering and mapping nanocavity emission via precision placement of DNA origami. Nature 2016, 535, 401-405.

(47) Minkov, M.; Savona, V. Automated optimization of photonic crystal slab cavities. Sci. Rep. 2014, 4, 5124.

(48) Oskooi, A. F.; Roundy, D.; Ibanescu, M.; Bermel, P.; Joannopoulos, J.; Johnson, S. G. Meep: A flexible free-software package for electromagnetic simulations by the FDTD method. Comput. Phys. Commun. 2010, 181, 687-702.

(49) Schülli, T. U.; Vastola, G.; Richard, M.-I.; Malachias, A.; Renaud, G.; Uhlík, F.; Montalenti, F.; Chen, G.; Miglio, L.; Schäffler, F.; Bauer, G. Enhanced Relaxation and Intermixing in Ge Islands Grown on PitPatterned Si(001) Substrates. Phys. Rev. Lett. 2009, 102, 025502.

(50) Pezzoli, F.; Stoffel, M.; Merdzhanova, T.; Rastelli, A.; Schmidt, O. G. Alloying and Strain Relaxation in SiGe Islands Grown on PitPatterned Si(001) Substrates Probed by Nanotomography. Nanoscale Res. Lett. 2009, 4, 1073-1077.

(51) Brehm, M.; Grydlik, M.; Groiss, H.; Hackl, F.; Schäffler, F.; Fromherz, T.; Bauer, G. The influence of a Si cap on self-organized SiGe islands and the underlying wetting layer. J. Appl. Phys. 2011, 109, 123505.

(52) Maleville, C.; Aspar, B.; Poumeyrol, T.; Moriceau, H.; Bruel, M.; Auberton-Hervè, A.; Barge, $\mathrm{T}$. Wafer bonding and $\mathrm{H}$-implantation mechanisms involved in the Smart-cut ${ }^{\circledR}$ technology. Mater. Sci. Eng., B 1997, 46, 14-19.

(53) Lo Savio, R.; Portalupi, S. L.; Gerace, D.; Shakoor, A.; Krauss, T. F.; O'Faolain, L.; Andreani, L. C.; Galli, M. Room-temperature emission at telecom wavelengths from silicon photonic crystal nanocavities. Appl. Phys. Lett. 2011, 98, 201106.

(54) Hackl, F.; Grydlik, M.; Brehm, M.; Groiss, H.; Schäffler, F.; Fromherz, T.; Bauer, G. Microphotoluminescence and perfect ordering of $\mathrm{SiGe}$ islands on pit-patterned $\mathrm{Si}(001)$ substrates. Nanotechnology 2011, 22, 165302.

(55) Kern, W. The Evolution of Silicon Wafer Cleaning Technology. J. Electrochem. Soc. 1990, 137, 1887-1892.

(56) Lichtenberger, H.; Mühlberger, M.; Schäffler, F. Transientenhanced $\mathrm{Si}$ diffusion on native-oxide-covered $\mathrm{Si}(001)$ nanostructures during vacuum annealing. Appl. Phys. Lett. 2003, 82, 3650.

(57) Brehm, M.; Montalenti, F.; Grydlik, M.; Vastola, G.; Lichtenberger, H.; Hrauda, N.; Beck, M. J.; Fromherz, T.; Schäffler, F.; Miglio, L.; Bauer, G. Key role of the wetting layer in revealing the hidden path of $\mathrm{Ge} / \mathrm{Si}(001)$ Stranski-Krastanow growth onset. Phys. Rev. B: Condens. Matter Mater. Phys. 2009, 80, 205321.

(58) Zhang, J. J.; Rastelli, A.; Schmidt, O. G.; Bauer, G. Role of the wetting layer for the SiGe Stranski-Krastanow island growth on planar and pit-patterned substrates. Semicond. Sci. Technol. 2011, 26, 014028.

(59) Ooka, Y.; Tetsumoto, T.; Fushimi, A.; Yoshiki, W.; Tanabe, T. CMOS compatible high- $Q$ photonic crystal nanocavity fabricated with photolithography on silicon photonic platform. Sci. Rep. 2015, 5, 11312.

(60) Galli, M.; Portalupi, S. L.; Belotti, M.; Andreani, L. C.; O'Faolain, L.; Krauss, T. F. Light scattering and Fano resonances in high-Q photonic crystal nanocavities. Appl. Phys. Lett. 2009, 94, 071101 .

(61) Hauke, N.; Lichtmannecker, S.; Zabel, T.; Laussy, F. P.; Laucht, A.; Kaniber, M.; Bougeard, D.; Abstreiter, G.; Finley, J. J.; Arakawa, Y. Correlation between emission intensity of self-assembled germanium islands and quality factor of silicon photonic crystal nanocavities. Phys. Rev. B: Condens. Matter Mater. Phys. 2011, 84.

(62) Hauke, N.; Zabel, T.; Müller, K.; Kaniber, M.; Laucht, A.; Bougeard, D.; Abstreiter, G.; Finley, J. J.; Arakawa, Y. Enhanced photoluminescence emission from two-dimensional silicon photonic crystal nanocavities. New J. Phys. 2010, 12, 053005.

(63) Brehm, M.; Suzuki, T.; Fromherz, T.; Zhong, Z.; Hrauda, N.; Hackl, F.; Stangl, J.; Schäffler, F.; Bauer, G. Combined structural and photoluminescence study of SiGe islands on Si substrates: comparison with realistic energy level calculations. New J. Phys. 2009, 11, 063021. 